\title{
The Abdominal Musculature and Cycling Performance
}

Meuwissen, T., Chandler, A., Swensen, T. Department of Exercise and Sport Sciences, Ithaca College, Ithaca, NY.

Purpose: Our purposes were to determine if abdominal power and endurance were related to anaerobic and aerobic cycling performance and to evaluate if abdominal fatigue effects the aforementioned cycling parameters. Methods: Twenty three college aged subjects had their age $(19.17 \pm 0.98 \mathrm{yrs})$, height $(170.41 \pm 7.54 \mathrm{~cm})$, and weight $(74.48 \pm$ $14.06 \mathrm{~kg}$ ) taken and completed the front abdominal power throw and ACSM Crunch test so we could evaluate their abdominal power and endurance, respectively; the tests were completed twice across $48 \mathrm{hr}$ to attenuate any learning effects. Twelve of the subjects completed the Wingate anaerobic power test on a Monark $834 \mathrm{E}$ ergometer set at $7.5 \%$ of body mass. The remaining 11 subjects completed a $3 \mathrm{~km}$ cycling time trail (TT), an aerobic cycling test, on an Expresso S3U virtual reality bike. Subjects completed familiarization, baseline, and performance trials for the cycling measures; immediately before the performance trials, subjects completed abdominal crunches to fatigue. All tests were preceded and followed by a warm-up and cool-down. Dependent $t$-tests were used to assess differences between baseline and performance cycling trails, whereas correlational analyses were used to evaluate the relationships between abdominal and cycling measures; a was set at 0.05. Results: Abdominal muscle fatigue significantly decreased mean anaerobic power $(p=0.000)$ and rate of fatigue $(p=0.004)$; the decrease in peak power approached significance $(p=0.088)$. Abdominal muscle fatigue didn't affect TT performance; however, after fatigue, abdominal power was significantly correlated to TT mean power and time ( $r=-0.708$ and 0.704 , respectively). No other significant correlations were found before or after fatigue between the abdominal and cycling measures. Conclusion: The data show that abdominal fatigue affects anaerobic cycling performance in our subject population; consequently, individuals may wish to avoid fatiguing abdominal exercise prior to anaerobic power tests or competitions that include anaerobic power elements. 